\title{
Atención a la salud maternoinfantil en Cuba: logros y desafíos
}

\author{
María Cecilia Santana Espinosa, ${ }^{1}$ Mercedes Esquivel Lauzurique, ${ }^{2}$ Violeta Regla \\ Herrera Alcázar, ${ }^{1}$ Berta Lidia Castro Pacheco, ${ }^{3}$ María del Carmen Machado \\ Lubián, ${ }^{4}$ Duniesky Cintra Cala, ${ }^{5}$ Roberto Tomás Álvarez Fumero ${ }^{6}$ y Beatriz \\ Marcheco Teruel
}

Forma de citar

Santana Espinosa MC, Esquivel Lauzurique M, Herrera Alcázar VR, Castro Pacheco BL, Machado Lubián MC, Cintra Cala D, et al. Atención a la salud maternoinfantil en Cuba: logros y desafíos. Rev Panam Salud Publica. 2018;42:e27. https://doi.org/10.26633/RPSP.2018.27

RESUMEN En Cuba, la atención a la salud maternoinfantil se sustenta en la prioridad que le otorga el Estado, la ejecución del Programa Nacional de Atención Materno Infantil y la garantía de acceso equitativo a los servicios de salud. Este artículo describe la experiencia cubana en este campo, así como sus principales logros, desafíos y lecciones aprendidas. Entre los resultados más relevantes hasta el 2015 se encuentran la reducción de la mortalidad infantil y del menor de 5 años a 4,3 y 5,7 fallecidos por 1000 nacidos vivos, respectivamente; supervivencia a los 5 años de 99,4\%; más de 10 controles prenatales por parto; 5,3\% de peso bajo al nacer; 99,9\% de partos institucionales; y ser el primer país en validar la eliminación de la transmisión vertical del VIH y la sífilis congénita. Los principales desafíos son aumentar la tasa de lactancia materna exclusiva; reducir la anemia por déficit de hierro en niños y gestantes y el sobrepeso infantil; prevenir los accidentes; y reducir la mortalidad materna, la tasa de fecundidad en las adolescentes y el aborto voluntario. Entre las lecciones aprendidas se destacan la prioridad que el Estado otorga a la salud, la conducción programática de la atención maternoinfantil, la garantía de cobertura universal, la recolección sistemática de información para la toma de decisiones, la integración de los sectores y la participación social en la salud. Sostener y mejorar los resultados alcanzados contribuirá al cumplimiento de la Agenda de Desarrollo Sostenible para el 2030.

Palabras clave

Salud materna; salud del niño; mortalidad materna; mortalidad infantil; Objetivos de Desarrollo del Milenio; Objetivos de Desarrollo Sostenible; Cuba.

\footnotetext{
Al triunfo de la Revolución Cubana en 1959, la salud en Cuba se caracterizaba por tasas elevadas de mortalidad infantil y materna (70 y 138 por 100000 nacidos vivos, respectivamente) como consecuencia del acceso limitado a los

Escuela Nacional de Salud Pública, La Habana, Cuba. Enviar la correspondencia a María Cecilia Santana Espinosa, cecilia.santana@infomed.sld.cu 2 Facultad de Ciencias Médicas Julio Trigo López, Universidad de Ciencias Médicas de La Habana, Cuba.

3 Hospital Pediátrico Universitario Juan Manuel Márquez, La Habana, Cuba.
}

servicios de salud, el elevado índice de analfabetismo, la escasa infraestructura sanitaria y la discriminación racial y de género, entre otros determinantes sociales (1-3). Las enfermedades diarreicas y respiratorias agudas, la desnutrición y

\footnotetext{
4 Instituto Nacional de Higiene, Epidemiología y Microbiología, La Habana, Cuba.

5 Organización Panamericana de la Salud, representación en Cuba, La Habana, Cuba.

6 Departamento de Atención Materno Infantil, Ministerio de Salud Pública, La Habana, Cuba.

7 Centro Nacional de Genética Médica, La Habana, Cuba.
}

las afecciones perinatales sobresalían como causas de muerte infantil, mientras que las madres morían por falta de atención a las complicaciones del parto y el aborto, y la enfermedad hipertensiva del embarazo. Solo el 10\% de la población infantil recibía atención pediátrica y menos del $60 \%$ de los partos ocurría en instituciones de salud (1-4).

Transformar esa situación requirió de la intervención del Estado, mediante la aplicación de nuevas políticas sociales y el sustento financiero estatal. De esta

Este es un artículo de acceso abierto distribuido bajo los términos de la licencia Creative Commons Attribution-NonCommercial-NoDerivs 3.0 IGO, que permite su uso, distribución y reproducción en cualquier medio, siempre que el trabajo original se cite de la manera adecuada. No se permiten modificaciones a los artículos ni su uso comercial. Al reproducir un artículo no debe haber ningún indicio de que la OPS o el artículo avalan a una organización o un producto específico. El uso del logo de la OPS no está permitido. Esta leyenda debe conservarse, junto con la URL original del artículo. 
forma se cumplió lo refrendado en el artículo 50 de la Constitución de la República, referente al derecho ciudadano a la salud y la obligación estatal de garantizarla, lo que se concreta mediante el Sistema Nacional de Salud (SNS). Este es un sistema que se caracteriza por ser único, gratuito y con acceso universal, cuya estrategia principal se centra en la atención primaria de salud (APS) y su línea general de desarrollo es la prevención. Ha contado, además, con una infraestructura y un capital humano y tecnológico en constante evolución. A ello se ha sumado la activa participación intersectorial y ciudadana, que se incrementa ante situaciones de crisis, como en los eventos hidrometeorológicos y las epidemias $(3,5,6)$.

Una estrategia fundamental para lograr resultados satisfactorios en la atención maternoinfantil fue la implementación del Programa Nacional de Atención Materno Infantil (PAMI) en 1983. El PAMI es una plataforma programática centralizada y dirigida por el Ministerio de Salud Pública (MINSAP) para planificar, organizar, aplicar y controlar en todo el país las acciones y las normativas relacionadas con la salud reproductiva, y de la infancia y la adolescencia, en correspondencia con el análisis de la situación de salud a nivel local y con hincapié en garantizar el acceso equitativo a la atención de salud (7).

El PAMI asumió las mejores experiencias desarrolladas durante las décadas de 1960 y 1970: el programa de la lucha contra la gastroenteritis (1962) (4), que desplazó esa afección del registro de las principales causas de muertes pediátricas; la primera campaña de vacunación antipoliomielítica (1962), que logró la eliminación sostenida de la enfermedad y sentó las bases para el Programa Nacional de Inmunización (1962); y la campaña masiva de vacunación contra la difteria, el tétanos y la tos ferina, también ese mismo año. A la campaña de vacunación contra el sarampión (1971), le siguieron otras acciones en la atención infantil que permitieron la eliminación del tétanos neonatal (1972), la difteria (1979), el síndrome de rubéola congénita (1989), la meningoencefalitis posparotiditis (1989), la rubéola (1995), así como la reducción de la morbilidad y la mortalidad de la enfermedad meningocócica (desde el 2002) y por Haemophilus influenzae tipo b (2003), la parotiditis (2004) y la hepatitis B $(2003)(4,8,9)$.
La prioridad de garantizar el parto institucional condujo en 1961 a la creación de los hospitales rurales con salones de parto en todo el país y al establecimiento en 1962 de los primeros hogares maternos. Estos son instituciones comunitarias cercanas a un hospital en las que ingresan las gestantes residentes en zonas de difícil acceso. Estas instituciones después se generalizaron y con el tiempo ampliaron sus funciones para atender también a mujeres con riesgos en cualquier etapa del embarazo. En los hogares maternos se materializa la práctica intersectorial y el apoyo comunitario, pues varios sectores contribuyen a su funcionamiento, como los de agricultura, deportes, cultura y educación, además de organizaciones de la sociedad civil como la Federación de Mujeres Cubanas (10-12). En el 2015 se notificaron en los hogares maternos 52,5 ingresos por cada 100 nacidos vivos (8).

En la década de 1960 era elevado el número de abortos clandestinos, que se realizaban en condiciones inadecuadas y por personal poco experimentado. En 1968, ante el reclamo de los derechos reproductivos de las mujeres y para contribuir a la disminución de la mortalidad, se institucionalizó el acceso a los servicios de aborto en los hospitales autorizados (11).

Estas y otras acciones han modificado sustancialmente la situación de salud de las mujeres y los recién nacidos. Véanse, por ejemplo, los indicadores registrados en el 2015 para las zonas montañosas del país, que son también las más remotas: 91\% de captación precoz del embarazo; índice de peso bajo al nacer de 4,7\%; y una tasa de mortalidad infantil de 3,8 por 1000 nacidos vivos, cifras todas inferiores a la media nacional (8).

Los propósitos del presente artículo son exponer la experiencia cubana de atención a la salud maternoinfantil, mostrar los resultados obtenidos hasta el 2015, identificar los desafíos para el cumplimiento de los Objetivos de Desarrollo Sostenible para el 2030 y presentar las principales lecciones aprendidas durante más de cinco décadas.

\section{ATENCIÓN A LA SALUD MATERNOINFANTIL}

En lo que respecta a la salud maternoinfantil, en los últimos años se ha hecho un llamado internacional a construir sistemas de salud resilientes que garanticen la cobertura sanitaria universal y ofrezcan una atención de buena calidad en todos los entornos $(13,14)$.

Desde 1959, en Cuba se trabaja sistemáticamente en acciones que armonizan con ese encargo, mediante los programas y las estrategias dirigidos a resolver o modificar los problemas de salud más relevantes tanto de las mujeres como de los niños y los adolescentes (cuadro 1).

\section{Atención a niños y adolescentes}

Es destacable el esfuerzo mantenido del SNS para brindar atención integral a los niños y los adolescentes mediante el desarrollo de la puericultura, que garantiza consultas planificadas desde la etapa de recién nacidos y posterior seguimiento hasta la adolescencia $(20,21)$. Se realizan más de 3 millones de consultas anuales a los niños sanos de entre 1 y 14 años (8) y acciones de promoción del desarrollo infantil temprano, que se proveen de manera integrada con el Programa Educa a Tu Hijo, del Ministerio de Educación. Estos son ejemplos de buenas prácticas en materia de intersectorialidad, que han obtenido el reconocimiento del Fondo de Naciones Unidas para la Infancia (UNICEF) (22).

La atención pediátrica dispone de protocolos de actuación para el seguimiento de las enfermedades transmisibles de la infancia, los cuales se ajustan en los escenarios de atención primaria y secundaria en forma de guías para el control de las enfermedades diarreicas y respiratorias agudas, los síndromes neurológicos infecciosos y las arbovirosis, entre otras $(2,3)$.

Para el control de las enfermedades crónicas se promueven estrategias específicas que llevan a cabo equipos interdisciplinarios organizados por territorios con el asesoramiento de expertos a nivel nacional. Las principales estrategias están dirigidas a la atención de niños que padecen de diabetes, asma, cardiopatías, afecciones neurológicas, insuficiencia renal crónica, enfermedades oncohematológicas y afecciones que requieran de cirugías de alta complejidad - como los trasplantes de órganos-. El propósito fundamental es proveer la supervivencia y una mejor calidad de vida a estos pacientes (3).

La implementación, en 1988, del programa de reducción de la incidencia del peso bajo al nacer ha contribuido al continuo descenso de este índice (figura 1). En la década de 1990 se produjo un 
CUADRO 1. Hitos en la atención a la salud maternoinfantil en Cuba, 1959-2015

\begin{tabular}{|c|c|}
\hline Período & Hecho relevante \\
\hline 1959-1969 & $\begin{array}{l}\text { - Programa de lucha contra la gastroenteritis } \\
\text { - Programa Nacional de Inmunización } \\
\text { - Programa de formación de los recursos humanos para la atención pediátrica, ginecológica } \\
\text { - } \text { Implemstétrica } \\
\text { - Creación de los salones de partos en los hospitales rurales }\end{array}$ \\
\hline 1970-1979 & $\begin{array}{l}\text { - Programa para la reducción de la mortalidad infantil } \\
\text { - Creación del Grupo Nacional de Pediatría y del Grupo Nacional de Ginecología y Obstetricia } \\
\text { - Inicio de los estudios antropométricos masivos y periódicos en los niños } \\
\text { - Creación de la especialidad de neonatología } \\
\text { - Programa para la reducción de la mortalidad materna } \\
\text { - Programa para la detección precoz del cáncer cervicouterino } \\
\text { - Publicación del Manual clínico de ginecología y obstetricia y del Manual clínico de pediatría }\end{array}$ \\
\hline 1980-1989 & $\begin{array}{l}\text { - Creación del Programa Nacional de Atención Materno Infantil } \\
\text { - Implementación del Plan del Médico y la Enfermera de la Familia } \\
\text { - Creación de la red de terapia intensiva pediátrica } \\
\text { - Creación del Cardiocentro Pediátrico William Soler y de la red cardiopediátrica } \\
\text { - Programa para la reducción del peso bajo al nacer } \\
\text { - Programa de diagnóstico prenatal de malformaciones congénitas } \\
\text { - Incorporación de tecnologías de diagnóstico prenatal }\end{array}$ \\
\hline 1990-1999 & $\begin{array}{l}\text { - Tamizaje de enfermedades metabólicas en los neonatos } \\
\text { - Programa de prevención de accidentes en los menores de } 20 \text { años } \\
\text { - Programa de atención a las enfermedades crónicas de la infancia } \\
\text { - Implementación de las consultas de ginecología infantojuvenil }\end{array}$ \\
\hline 2000-2009 & $\begin{array}{l}\text { - Programa de atención integral al adolescente } \\
\text { - Perfeccionamiento de la atención a los grupos vulnerables, las personas con } \\
\text { discapacidades y las víctimas de desastres naturales } \\
\text { - Fortalecimiento de los servicios de puericultura } \\
\text { - Fortalecimiento tecnológico y de capacitación de los recursos humanos dedicados a la } \\
\text { terapia intensiva y neonatal }\end{array}$ \\
\hline 2010-2015 & $\begin{array}{l}\text { - Actualización del Programa de Reducción de la Morbilidad y Mortalidad Materna } \\
\text { - } \quad \text { Creación de los bancos de leche humana } \\
\text { - Implementación del protocolo de ventilación mecánica domiciliaria }\end{array}$ \\
\hline
\end{tabular}

Fuente: Elaboración de los autores a partir de las referencias 1-4, 9, 11, 12, 15-31.

FIGURA 1. Índice de peso bajo al nacer. Cuba, 1975-2015

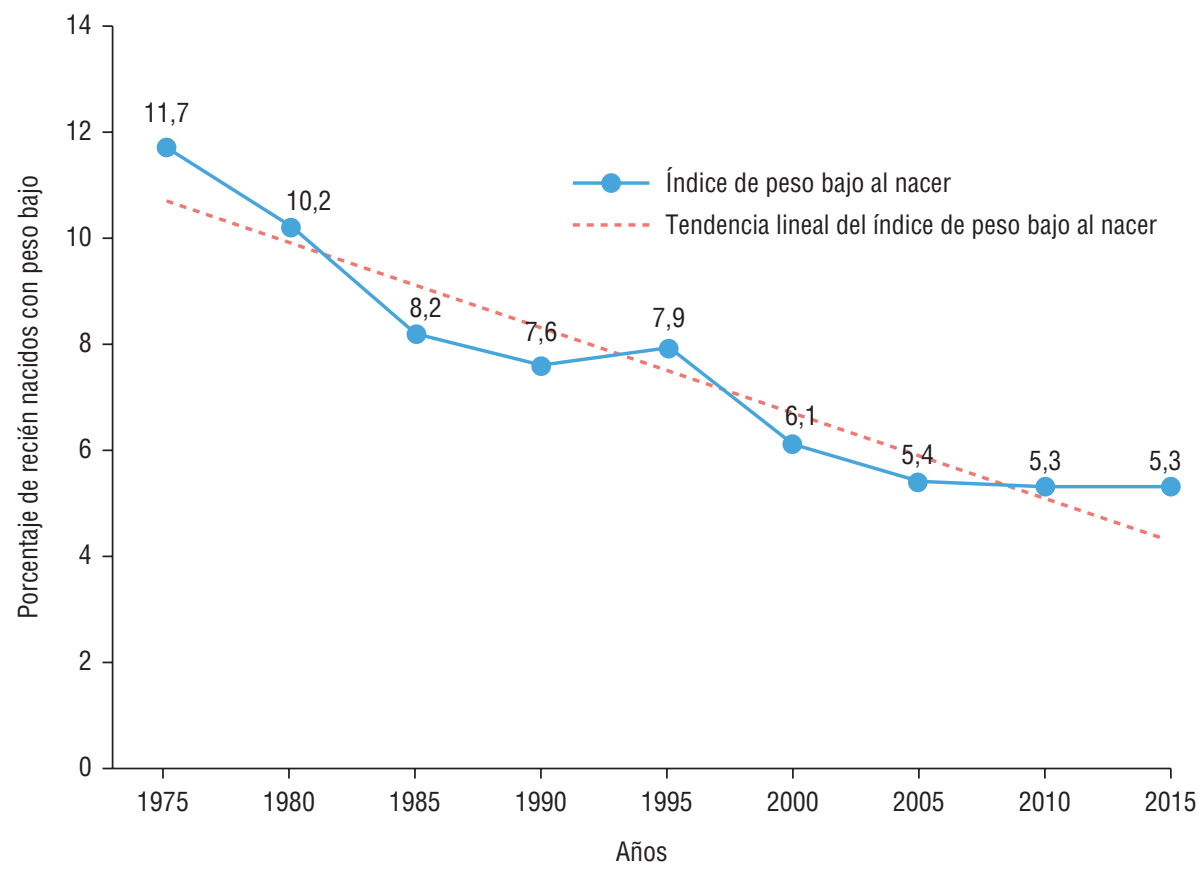

Fuente: Elaboración de los autores a partir de la referencia 8. incremento de este indicador debido a la agudización de las dificultades económicas del país (23), lo que justificó la actualización del programa. A pesar de las bajas tasas actuales, se mantiene una atención especial al desarrollo de la perinatología (12), ya que se considera que nacer con un peso inferior a 1000 gramos representa un problema para la supervivencia inmediata y la calidad de vida posterior.

Entre las principales estrategias dirigidas a la atención del recién nacido se encuentran las pruebas de tamizaje metabólico para la detección precoz del hipotiroidismo congénito (801 casos detectados en 3350373 muestras analizadas); la fenilcetonuria (20 en 1055575 muestras); la hiperplasia suprarrenal congénita (45 en 780 771); el déficit de biotinidasa (5 en 759 935) y la galactosemia (7 en 723 182), todas realizadas con tecnologías desarrolladas en Cuba (24).

Asimismo, existen redes de salud de carácter multidisciplinario que tienen amplia participación intersectorial y social, entre las que se encuentran:

- El Centro Nacional de Genética Médica, creado en 1980, encargado de conducir metodológicamente el Programa Nacional de Diagnóstico, Manejo y Prevención de Enfermedades Genéticas y Defectos Congénitos mediante una red nacional de 184 centros y servicios, y un valioso capital humano especializado, tanto en genética clínica como comunitaria, actúa desde la APS. Este sistema ha contribuido a reducir la mortalidad infantil por anomalías congénitas en más de $70 \%$ (25).

- La red de salas de terapia intensiva pediátrica, creada en 1982 en respuesta a la epidemia de dengue hemorrágico que cobró la vida de más de 100 niños, cuenta con 32 salas distribuidas en todas las provincias. Desde 1985 se conservan los registros de movimiento hospitalario de estos servicios en la Dirección Nacional de Registros Médicos del MINSAP. En el 2016 se notificaron 11434 pacientes ingresados, para un índice ocupacional promedio de 58,3, una estadía promedio de 4,7 días y una tasa de mortalidad bruta de 3,6. Estos indicadores reflejan un mejoramiento del servicio de atención al niño grave $(8,26)$.

- El Cardiocentro Pediátrico William Soler, fundado en 1986, coordina la 
red cardiopediátrica nacional y garantiza la atención especializada y continua desde la etapa prenatal hasta la vida adulta. Para ello cuenta con un equipo integrado por cardiólogos, cirujanos, anestesiólogos, personal de enfermería, pediatras y especialistas en medicina general integral, radicados en todas las provincias y los municipios, y que garantiza el diagnóstico precoz y el tratamiento médico, quirúrgico y de rehabilitación de los niños con cardiopatías (27).

Otras acciones importantes son la presencia de la madre acompañante en los hospitales pediátricos, la categorización de los hospitales Amigos de la Madre y el Niño, y la puesta en funcionamiento de los bancos de leche humana $(1,28)$.

Los accidentes son la primera causa de muerte en los grupos de 1 a $4 ; 5$ a $14 ;$ y 10 a 19 años, con tasas respectivas de 0,6; 6,1 y 8,1 por 100000 habitantes de esos grupos de edad, respectivamente; mientras que en los menores de 1 año, esta es la cuarta causa de muerte más frecuente (0,1 por 1000 nacidos vivos). Por esta razón, existe un programa de prevención de accidentes en los menores de 20 años, cuyas acciones fundamentales son de promoción y prevención $(8,29)$.

\section{Atención a la mujer}

La atención prenatal cuenta con una metodología aplicada básicamente desde la APS, lo que garantiza el acceso y la cobertura universales, y comprende acciones como la captación precoz de las embarazadas, los estudios iniciales para detectar infecciones, enfermedades crónicas, riesgo genético y otros trastornos relacionados con el embarazo, además de la atención estomatológica, el aporte de suplementos vitamínicos y dietéticos, y la atención especializada $(7,30)$.

El aborto es un problema de salud reproductiva. En Cuba no se reconoce como método anticonceptivo; sin embargo, se mantiene una elevada frecuencia de la práctica del aborto ante un embarazo no deseado (30 por cada 1000 mujeres de 12 a 49 años de edad), en particular entre las adolescentes y las jóvenes (8). Para disminuir este indicador se llevan a cabo acciones promocionales y educativas, sobre todo desde la APS y con la participación de las organizaciones de la sociedad civil y los medios de comunicación.
La tasa de mortalidad materna se ha reducido (figura 2), pero desde 1990 mantiene un comportamiento oscilante con cifras cercanas a 40 por 100000 nacidos vivos. Las causas de mayor incidencia son las hemorragias graves (posparto o por embarazos ectópicos), las infecciones, los abortos espontáneos y la hipertensión gestacional (preeclampsia) $(8,11)$. Esto motivó la actualización del Programa de Reducción de la Morbilidad y Mortalidad Materna en el año 2012 (31).

Así, este programa incorporó nuevas actividades en materia de calidad en los servicios para la atención de emergencias y eventualidades relacionadas con el embarazo, el parto y el puerperio, para lo que se ha requerido la introducción de tecnologías de avanzada y la continua calificación del personal (31).

Para garantizar la salud reproductiva se ejecutan programas dirigidos a la atención a la pareja infértil y la detección precoz de cáncer cervicouterino, de mama y otros. También son relevantes las acciones para la planificación familiar, cuya estrategia principal va dirigida al riesgo reproductivo preconcepcional, orientada a la educación y la información de las parejas sobre el uso profiláctico del ácido fólico, así como la detección y la compensación de las

\section{FIGURA 2. Tasa de mortalidad materna. Cuba, 1970-2015}

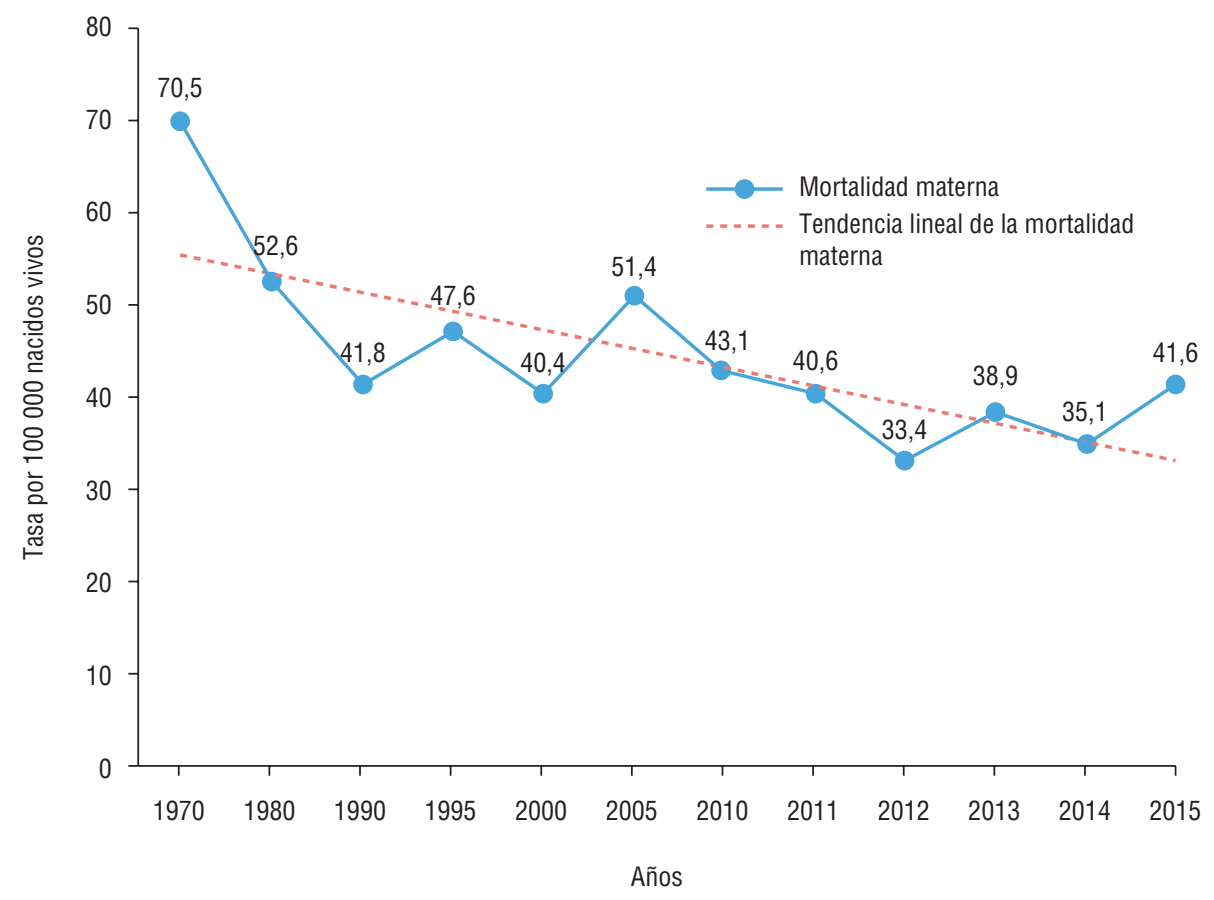

Fuente: Elaboración de los autores a partir de la referencia 8. enfermedades o los factores de riesgo antes de la concepción, sobre todo los relacionados con las complicaciones maternas $(7,30)$.

Se preconiza el empleo oportuno de métodos anticonceptivos - transitorios o permanentes-, que se ofrecen de forma gratuita, incluida la opción de la anticoncepción de emergencia, la cual está disponible en todos los consultorios del Programa del Médico y la Enfermera de la Familia. Cuba ha alcanzado $77,1 \%$ de cobertura anticoncepcional total, aunque existe insatisfacción en cuanto a la estabilidad de la cobertura de los métodos hormonales (8).

En respuesta a la situación epidemiológica relacionada con las infecciones por arbovirus, se ha implementado una estrategia de vigilancia y control, con un plan de acción para la mujer en edad fértil y para la pesquisa y la prevención de daños genéticos durante el embarazo (32).

Las estrategias, las normativas y los programas establecidos en beneficio de la salud maternoinfantil requieren de una constante renovación, con la introducción de los nuevos conocimientos y tecnologías, sobre la base de las evidencias científicamente probadas; de ahí la importancia de las investigaciones y la aplicación de sus resultados. 


\section{INVESTIGACIONES PARA LA SALUD MATERNOINFANTIL}

Algunas investigaciones se ejecutan de forma sistemática, con diseños metodológicos similares, lo que permite la comparación entre varios estudios y la identificación de las tendencias en indicadores que no forman parte del sistema de registros médicos y las estadísticas de salud, pero que son muy útiles en el campo de la salud maternoinfantil.

Este es el caso de los estudios poblacionales periódicos sobre crecimiento y desarrollo de los niños y los adolescentes —de 0 a 18 años-, realizados aproximadamente cada 10 años. Estas investigaciones han proporcionado los valores de referencia para la vigilancia del crecimiento infantil en la práctica asistencial y el monitoreo de la tendencia secular del crecimiento (15). También han contribuido a este conocimiento las Encuestas de Indicadores Múltiples por Conglomerados (MICS), realizadas en colaboración con la UNICEF (33). Estas se ejecutan cada 4 años para conocer las tendencias en la alimentación de los niños pequeños, la atención de las enfermedades diarreicas y respiratorias, la salud reproductiva, la protección infantil, la prevalencia de la infección por el virus de la inmunodeficiencia humana y el sida (VIH/sida) y el comportamiento sexual.

Otros estudios destacados han sido la investigación perinatal realizada a todos los nacidos vivos en la primera semana de marzo de 1973 y el seguimiento de esa cohorte durante más de 15 años; los trabajos sobre mortalidad materna, enfermedades diarreicas agudas, anemia, discapacidad, accidentes y la experiencia cubana en la atención a la salud infantil 1959-2006, en colaboración con el Departamento de Salud del Niño y el Adolescente de la Organización Mundial de la Salud (OMS) $(2,3,11,29,34)$.

\section{LOGROS Y DESAFÍOS EN LA ATENCIÓN A LA SALUD MATERNOINFANTIL}

Con las acciones implementadas durante más de cinco décadas se han alcanzado indicadores de salud maternoinfantil favorables, a pesar de las limitaciones económicas y de recursos $(23,35)$. Se destaca la disminución de las tasas de mortalidad infantil y de los menores de 5 años a 4,3 y 5,7 por 1000 nacidos vivos, respectivamente (figura 3), que ubican a Cuba, junto a Canadá, como los países con las cifras más bajas en la Región de las Américas (36). El porcentaje de niños supervivientes a los 5 años de edad es de $99,4 \%$ (8).

El desarrollo de la genética clínica y comunitaria, la formación y el perfeccionamiento de los recursos humanos, la tendencia secular positiva del crecimiento de los niños, el incremento en la cantidad y la calidad de los controles de salud, la elevada cobertura de

FIGURA 3. Tasa de mortalidad infantil y tasa de mortalidad en menores de 5 años. Cuba, 1970-2015

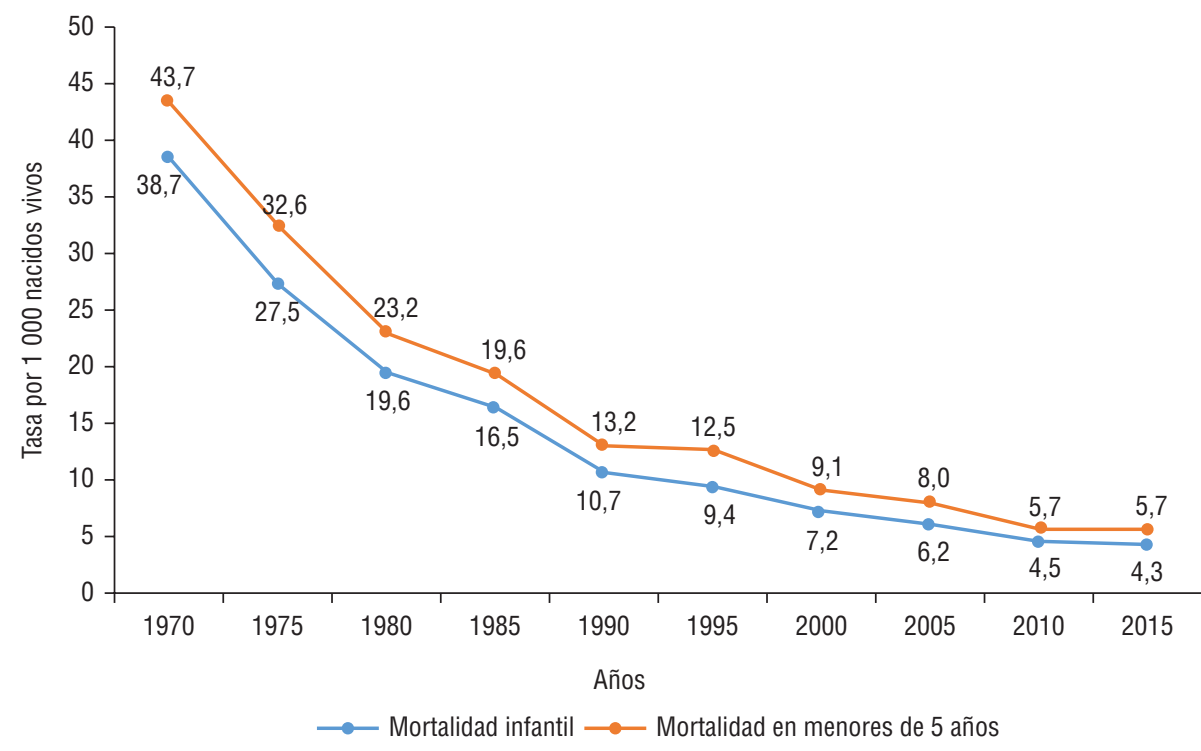

Fuente: Elaboración de los autores a partir de la referencia 8.

vacunación y la reducción de la morbilidad y la mortalidad por enfermedades infecciosas han influido decisivamente en esos resultados.

En el 2015, Cuba se convirtió en el primer país del mundo en validar la eliminación de la transmisión vertical del VIH y la sífilis congénita (37).

A pesar de estos indicadores relevantes, Cuba no cumplió tres de los Objetivos de Desarrollo del Milenio (ODM) (36) (cuadro 2):

- ODM4: Reducir la mortalidad de los niños menores de 5 años. No se alcanzó la reducción propuesta porque las tasas en 1990 ya eran muy bajas. Las principales causas de muerte en los menores de 1 año fueron las afecciones perinatales, las malformaciones congénitas, y la influenza y la neumonía (tasas de 2,1; 0,9 y 0,3 por 1000 nacidos vivos, respectivamente), mientras que en los niños de 1 a 4 años fueron los accidentes, los tumores malignos y las malformaciones congénitas (tasas de 0,8; 0,7 y 0,3 por 10000 habitantes de ese grupo de edad, respectivamente) (8). La cobertura de inmunización contra el sarampión está cumplida. Las demás vacunas, aplicadas según el Programa Nacional de Inmunización, tienen una cobertura superior a 95\% (8).

- ODM5: Mejorar la salud materna. Este objetivo no se alcanzó totalmente, pues no se redujo la mortalidad materna. Las principales causas directas de muerte han sido las hemorragias, las complicaciones tromboembólicas y la sepsis, mientras que las indirectas se relacionaron con los aparatos circulatorio y respiratorio (8). La tasa de fecundidad en las adolescentes disminuyó, aunque continúa siendo un problema de salud por sus implicaciones médicas y sociales. La cobertura de atención prenatal es elevada, con más de 10 controles por parto; y se garantiza el parto institucional con atención especializada. En relación con la planificación familiar, se alcanza una adecuada cobertura anticoncepcional $(8,33)$.

- ODM6: Combatir el VIH/sida, el paludismo y otras enfermedades. La prevalencia de $\mathrm{VIH} /$ sida se mantiene en cifras inferiores a $0,1 \%$. Se garantiza la oferta de condones (incluido el suministro gratuito para los grupos 
CUADRO 2. Cumplimiento de los Objetivos de Desarrollo del Milenio (ODM). Cuba, 1990-2015a

\begin{tabular}{|c|c|c|c|c|}
\hline ODM y meta & Indicador & Cifra y año de inicio & Cifra y año de corte & Resultado (\%) \\
\hline \multicolumn{5}{|l|}{$\begin{array}{l}\text { A. Indicadores relacionados directamente con la } \\
\text { ODM 4. Reducir la mortalidad de los niños } \\
\text { menores de } 5 \text { años }\end{array}$} \\
\hline \multirow{3}{*}{$\begin{array}{l}\text { Meta } 4 \text { A. Reducir en las dos terceras partes, } \\
\text { entre } 1990 \text { y el } 2015 \text {, la mortalidad de los } \\
\text { niños menores de } 5 \text { años }\end{array}$} & $\begin{array}{l}\text { 4.1. Tasa de mortalidad de los niños menores } \\
\text { de } 5 \text { años por } 1000 \text { nacidos vivos }\end{array}$ & 13,2 en 1990 & 5,7 en el 2015 & Reducción: 56,8 \\
\hline & $\begin{array}{l}\text { 4.2. Tasa de mortalidad infantil por } \\
1000 \text { nacidos vivos }\end{array}$ & 10,7 en 1990 & 4,3 en el 2015 & Reducción: 59,8 \\
\hline & $\begin{array}{l}\text { 4.3. Inmunización contra el sarampión (\% de } \\
\text { cobertura)a }\end{array}$ & 94 en 1990 & 100 en el 2015 & Aumento: 6,4 \\
\hline \multicolumn{5}{|l|}{ ODM 5. Mejorar la salud materna } \\
\hline \multirow[t]{2}{*}{$\begin{array}{l}\text { Meta } 5 \text { A. Reducir, entre } 1990 \text { y el } 2015 \text {, la } \\
\text { mortalidad materna en tres cuartas partes }\end{array}$} & $\begin{array}{l}\text { 5.1. Razón de mortalidad materna por } \\
100000 \text { nacidos vivos }\end{array}$ & 41,8 en 1990 & 41,6 en el 2015 & Reducción: 0,5 \\
\hline & $\begin{array}{l}\text { 5.2. Proporción de partos con asistencia de } \\
\text { personal de salud calificado }(\%)^{\mathrm{a}}\end{array}$ & 99,8 en 1990 & 99,9 en el 2015 & Aumento: 0,1 \\
\hline \multirow[t]{3}{*}{$\begin{array}{l}\text { Meta 5B. Lograr para el } 2015 \text { el acceso } \\
\text { universal a la salud reproductiva }\end{array}$} & $\begin{array}{l}\text { 5.4. Tasa de fecundidad en adolescentes por } \\
1000 \text { mujeres de la edada }\end{array}$ & 77,5 en 1990 & 52,5 en el 2015 & Reducción: 32,3 \\
\hline & $\begin{array}{l}\text { 5.5. Cobertura de la atención prenatal } \\
\left(4 \text { consultas } 0 \text { más) }(\%)^{\mathrm{a}}\right.\end{array}$ & 11,8 en 1990 & 15,4 en el 2015 & Aumento: 30,5 \\
\hline & $\begin{array}{l}\text { 5.6. Necesidades insatisfechas en materia de } \\
\text { planificación familiar }(\%)^{a}\end{array}$ & 39,1 en 1990 & 8,0 en el 2014 & Reducción: 79,5 \\
\hline \multicolumn{5}{|c|}{ ODM 6. Combatir el VIH/sida, el paludismo y otras enfermedades } \\
\hline \multirow{3}{*}{$\begin{array}{l}\text { Meta } 6 \text { A. Haber detenido y comenzado a } \\
\text { reducir, para el año 2015, la propagación del } \\
\text { VIH/sida }\end{array}$} & $\begin{array}{l}\text { 6.1. Prevalencia de } \mathrm{VIH} / \text { sida entre las } \\
\text { personas de } 15 \text { a } 24 \text { años (\%) }\end{array}$ & 0,05 en el 2005 & 0,09 en el 2015 & Aumento: 80,0 \\
\hline & $\begin{array}{l}\text { 6.2. Uso de condones en la última relación } \\
\text { sexual de alto riesgo }(\%)^{\mathrm{a}}\end{array}$ & 7,2 en 1995 & †: 66,0 en el 2012 & Aumento: 816,7 \\
\hline & $\begin{array}{l}\text { 6.3. Proporción de la población de } 15 \text { a } \\
24 \text { años que tiene conocimientos amplios y } \\
\text { correctos sobre el VIH/sida }(\%)^{\mathrm{a}}\end{array}$ & 26 en 1995 & $\begin{array}{l}+: \text { : } 60,9 \text { ふ }: 58,6 \text { en el } \\
2014\end{array}$ & $\begin{array}{c}\text { Aumento: } \\
\text { o: } 134,2 \text { đ: } 125,4 \text { en el } \\
2014\end{array}$ \\
\hline $\begin{array}{l}\text { Meta 6B. Lograr para el año } 2010 \text { el acceso } \\
\text { universal al tratamiento del VIH/sida de todas } \\
\text { las personas que lo necesiten }\end{array}$ & $\begin{array}{l}\text { 6.5. Proporción de la población portadora del } \\
\text { VIH con infección avanzada que tiene acceso } \\
\text { a los medicamentos antirretrovirales }(\%)^{\mathrm{a}}\end{array}$ & 38,7 en el 2000 & 100,0 en el 2015 & Aumento: 158,4 \\
\hline \multicolumn{5}{|c|}{$\begin{array}{l}\text { ODM 1. Erradicar la pobreza extrema y el } \\
\text { hambre }\end{array}$} \\
\hline $\begin{array}{l}\text { Meta 1C. Reducir a la mitad, entre } 1990 \text { y el } \\
2015 \text {, el porcentaje de personas que padecen } \\
\text { hambre }\end{array}$ & $\begin{array}{l}\text { 1.8. Proporción de niños menores de } 5 \text { años } \\
\text { con peso inferior al estándar }(\%)^{\mathrm{a}}\end{array}$ & 6,1 en 1995 & 4,6 en el 2012 & Reducción: 24,6 \\
\hline \multicolumn{5}{|l|}{$\begin{array}{l}\text { ODM 7. Garantizar la sostenibilidad del medio } \\
\text { ambiente }\end{array}$} \\
\hline \multirow{2}{*}{$\begin{array}{l}\text { Meta } 7 \text { C. Reducir a la mitad, para el año } \\
2015 \text {, el porcentaje de personas sin acceso } \\
\text { sostenible al agua potable y a los servicios } \\
\text { básicos de saneamiento }\end{array}$} & $\begin{array}{l}\text { 7.8. Proporción de la población que utiliza } \\
\text { fuentes mejoradas de abastecimiento de agua } \\
\text { potable }(\%)^{\mathrm{a}}\end{array}$ & 78,2 en 1990 & 94,2 en el 2014 & Aumento: 20,5 \\
\hline & $\begin{array}{l}\text { 7.9. Proporción de la población que utiliza } \\
\text { servicios de saneamiento mejorados }(\%)^{\mathrm{a}}\end{array}$ & 88,7 en 1990 & 90,7 en el 2014 & Aumento: 2,3 \\
\hline
\end{tabular}

andicador ODM cumplido.

Nota: VIH: virus de la inmunodeficiencia humana.

Fuente: Elaboración de los autores a partir de las referencias 8, 36, 39.

de riesgo) y ha aumentado la proporción de la población de 15 a 24 años con conocimientos amplios y correctos sobre el VIH/sida (38). El 100\% de la población portadora o enferma de sida tiene acceso gratuito a los medicamentos antirretrovirales.

Entre las metas e indicadores de los ODM1 y ODM7, los avances se reflejan en las cifras muy bajas de niños menores de 5 años con un peso inferior al estándar y también en el comportamiento positivo del índice de peso bajo al nacer (8), así como el incremento de la proporción de población con acceso a las fuentes mejoradas de agua potable y los servicios básicos de saneamiento (36).

Con el inicio del 2016 entraron en vigor los Objetivos de Desarrollo Sostenible (ODS) de la Agenda 2030 (39). Los preceptos de desarrollo para Cuba hasta ese año coinciden en varios aspectos con los ODS y sus metas, de modo que estos desafíos continuarán formando parte de las políticas y los programas aprobados para el país.

Hoy, en Cuba se han logrado (total o parcialmente) algunas de las metas relacionadas con la salud maternoinfantil que propone la Agenda 2030. Entre ellas las relacionadas con las tasas de mortalidad neonatal y de niños menores de 5 años (meta 3.2), la prevalencia de emaciación y retardo del crecimiento en los niños preescolares (meta 2.2), el acceso universal a la 


\section{CUADRO 3. Lecciones aprendidas}

\begin{tabular}{|c|c|}
\hline $\begin{array}{l}\text { Factores que han contribuido } \\
\text { a la buena atención } \\
\text { maternoinfantil }\end{array}$ & Resultado \\
\hline Voluntad política & $\begin{array}{l}\text { La prioridad que el Estado le otorga a la salud ha posibilitado la elaboración y el } \\
\text { financiamiento de los programas necesarios para resolver o modificar los } \\
\text { problemas de salud más relevantes }\end{array}$ \\
\hline $\begin{array}{l}\text { Dirección centralizada de la } \\
\text { salud }\end{array}$ & $\begin{array}{l}\text { La conducción programática mediante el Programa Nacional de Atención Materno } \\
\text { Infantil ha propiciado los indicadores favorables de salud alcanzados en las } \\
\text { mujeres, los niños y los adolescentes }\end{array}$ \\
\hline $\begin{array}{l}\text { Resiliencia del sistema de } \\
\text { salud }\end{array}$ & $\begin{array}{l}\text { Ha brindado cobertura universal, equidad y preparación para el enfrentamiento a } \\
\text { emergencias }\end{array}$ \\
\hline Vigilancia de salud & $\begin{array}{l}\text { Ha estado dirigida a la recolección sistemática de datos para el análisis y la } \\
\text { interpretación de eventos específicos como base para la toma de decisiones } \\
\text { informadas }\end{array}$ \\
\hline Intersectorialidad & $\begin{array}{l}\text { Se ha logrado la integración de los sectores de la sociedad en las actividades que } \\
\text { se desarrollan para mejorar la salud de las madres, los niños y los adolescentes }\end{array}$ \\
\hline Participación social & $\begin{array}{l}\text { La contribución popular ha sido decisiva; está vinculada a la voluntad política y ha } \\
\text { sido notoria durante las etapas de crisis }\end{array}$ \\
\hline $\begin{array}{l}\text { Desarrollo de ambientes } \\
\text { protectores }\end{array}$ & $\begin{array}{l}\text { Ha abarcado las acciones intersectoriales ejecutadas para la provisión de agua } \\
\text { potable y de los servicios básicos de saneamiento y protección social }\end{array}$ \\
\hline $\begin{array}{l}\text { Utilización de las evidencias } \\
\text { científicas }\end{array}$ & $\begin{array}{l}\text { Ha permitido la selección de las intervenciones más eficaces en la búsqueda de } \\
\text { nuevas soluciones }\end{array}$ \\
\hline
\end{tabular}

Fuente: Elaboración de los autores.

salud sexual y reproductiva (meta 3.7) y la cobertura sanitaria universal (meta 3.8). La tasa de mortalidad materna, aunque cumple ampliamente la meta mundial de 70 por 100000 nacidos vivos (meta 3.1), es aún motivo de insatisfacción para las autoridades sanitarias cubanas. necen desafíos que requieren de un seguimiento priorizado para alcanzar los ODS:

- Aumentar la tasa de lactancia mater-
Junto a los logros alcanzados, permana exclusiva, de la que solo se beneficia en la actualidad 1 de cada 3 lactantes menores de 6 meses (33).

- Reducir los problemas nutricionales que persisten, como la prevalencia de anemia ligera por déficit de hierro en 1 de cada 5 gestantes en el tercer trimestre del embarazo y en 1 de cada 3 niños de 6 a 35 meses; además, detener y reducir el incremento del sobrepeso infantil, que ha mantenido una tendencia ascendente en las últimas décadas (22).

- Fortalecer las acciones de prevención de accidentes (29).

- Reducir la mortalidad materna por las principales causas (8).

- Reducir la tasa de fecundidad en las adolescentes y el empleo del aborto

\section{REFERENCIAS}

1. Valdés Lazo F. Práctica pediátrica en Cuba. En: De la Torre Montejo E, Pelayo GonzálezPosada EJ, ed. Pediatría. P1 T1. La Habana: Editorial Ciencias Médicas; 2006.

2. Riverón Corteguera RL. Estrategias para reducir la mortalidad infantil, Cuba 19591999. Rev Cubana Pediatr. 2000;72(3): 147-64. Disponible en: http://scielo.sld. $\mathrm{cu} /$ scielo.php? script=sci_arttext\&pid= S0034-75312000000300001 Acceso el 19 de agosto de 2017.

3. Castro Pacheco BL, Cuéllar Álvarez R, Ibargollen Negrín L, Esquivel Lauzurique M, Machado Lubián MC, Martínez Corredera V. Cuban experience in child health care. 1959-2006. La Habana: Editora Política; 2010. Disponible en: http://ipaworld.org/uploadedbyfck/Report $\% 20$ on $\%$ 20Child $\% 20$ Health $\% 20$ in $\% 20$ Cuba $\% 20$ by $\% 20 J$ ose $\% 20$ Martines_EXPERIENCIA \%
20CUBANA \%20PARA \%20EDITORA $\% 20$ POLITICA \% $20 \% 28$ ENGLISH $\% 20$ VERSION\%29.pdf Acceso el 19 de agosto de 2017.

4. Riverón Corteguera RL, Mena Miranda VR, González Fernández MA. Morbilidad y mortalidad por enfermedades infecciosas intestinales (001-009). Cuba, 1980-1999. Rev Cubana Pediatr. 2000;72(2):72-80. Disponible en: http://scielo.sld.cu/pdf/ ped/v72n2/ped02200.pdf Acceso el 10 de septiembre de 2017.

5. Constitución de la República de Cuba. Capítulo VII. Derechos, deberes y garantías fundamentales. Art 50. La Habana: Ministerio de Justicia; 1976. Disponible en: http:/ / www.cuba.cu/gobierno/cuba. htm Acceso el 18 de septiembre de 2017.

6. Castell-Florit Serrate P, Díaz Llanes G, Serrano Patten A, Bayarre Vea HD, Rojas voluntario como método contraceptivo (8).

- Reducir las necesidades insatisfechas en planificación familiar (33).

- Fortalecer las actividades dirigidas a reducir el riesgo preconcepcional $(11,31)$.

\section{LECCIONES APRENDIDAS}

Entre las lecciones aprendidas en más de 50 años se destaca el reconocimiento de la importancia de los factores que han contribuido a una adecuada atención maternoinfantil (cuadro 3).

\section{CONCLUSIONES}

Las políticas, las estrategias y las normativas desarrolladas por el SNS durante más de cinco décadas han influido favorablemente en los principales indicadores de salud maternoinfantil. De esta experiencia se derivan logros, desafíos y lecciones aprendidas que podrían adaptarse a otros contextos para alcanzar resultados similares.

Sostener y mejorar estos resultados son retos para cumplir la Agenda de Desarrollo Sostenible para el 2030.

\section{Conflicto de intereses. Ninguno.}

Declaración. Las opiniones expresadas en este manuscrito son responsabilidad de los autores y no reflejan necesariamente los criterios ni la política de la Revista Panamericana de Salud Pública / Pan American Journal of PublicHealth o de la Organización Panamericana de la Salud.
Hernández KI, Figueroa Cruz M, et al. Intersectorialidad en salud. Fundamentos y aplicaciones. La Habana: Editorial Ciencias Médicas; 2010.

7. Cuba, Ministerio de Salud Pública, Departamento Nacional de Salud Materno Infantil. Programa Nacional de Atención Materno Infantil. La Habana: MINSAP; 1983.

8. Cuba, Ministerio de Salud Pública, Dirección Nacional de Registros Médicos y Estadísticas de Salud. Anuario Estadístico de Salud (1995-2016). Disponible en: http:/ / bvscuba.sld.cu/anuario-estadistico-de-cuba / Acceso el 2 de junio de 2017.

9. Reed G, Galindo MA. Cuba's National Immunization Program. MEDICC Rev. 2007;9(1):5-7. Disponible en: http://www. medicc.org/mediccreview/index.php? issue $=6 \&$ id $=56 \& \mathrm{a}=$ vahtml Acceso el 6 de agosto de 2017. 
10. Delgado G. Los hogares maternos: su fundación en Cuba y objetivos propuestos desde su creación. Cuad Hist Salud Publica. 2004;95. Disponible en: http:// scielo.sld.cu/scielo.php?script=sci_arttext\& pid=S0045-91782004000100016\&lng=es Acceso el 6 de agosto de 2017.

11. Cabezas Cruz E. Evolución de la mortalidad materna en Cuba. Rev Cubana Salud Publica. 2006;32(1). Disponible en: http:/ / scielo.sld.cu/scielo.php?script=sci_arttext\& pid=S0864-34662006000100005 Acceso el 6 de agosto de 2017.

12. Gorry C. Lowering infant mortality in Cuba: interview to Fernando Domínguez MD PhD. MEDICC Rev. 2015;17(1):11-3. Disponible en: http:/ / www.scielosp.org/ pdf/medicc/v17n1/1555-7960-medicc-1701-0011.pdf Acceso el 6 de agosto de 2017.

13. Todas las Mujeres Todos los Niños. Estrategia Mundial para la Mujer, el Niño y el Adolescente (2016-2030). Nueva York: Naciones Unidas; 2015. Disponible en: http: / / www.everywomaneverychild. org / wp-content/uploads / 2017/10/ EWEC_GSUpdate_Brochure_ES_2017_ web.pdf Acceso el 6 de junio de 2017.

14. Lombardi J. Gaining momentum: advancing early childhood development 2016 [editorial]. Early Childhood Matters. 2016;(125):6-10. Disponible en: https:// bernardvanleer.org / app / uploads / 2016/06/Early-Childhood-Matters-2016. pdf Acceso el 21 de marzo de 2017.

15. Esquivel M, Jiménez S. Cambios en el crecimiento de los niños cubanos. Seguimiento y acciones. Arch Latinoamericanos Nutr. 2015;65(1 supl). Disponible en: http:// www.alanrevista.org/ediciones / 2015/ suplemento-1/art-242/\# Acceso el 3 de abril de 2017 .

16. Cabezas Cruz E, Camacho Martínez T, Santana Martínez A, Borrajero Martínez I, Aguilar Vela de Oro F, Romero Pérez T, et al. Programa Diagnóstico Precoz del Cáncer del Cuello del Útero. La Habana: Ministerio de Salud Pública; 1999. Disponible en: http://files.sld.cu/sida/ files/2012/01/prog-detec-canc-cervicout. pdf Acceso el 3 de septiembre de 2017.

17. Lugones Botell M, Pedroso Hernández P, Perera Boza O, Acosta Jiménez M. La consulta de ginecología infanto-juvenil en función de la educación sexual. Rev Cubana Med Gen Integr. 1999;15(2): 184-90. Disponible en: http://scielo.sld. cu/scielo.php?script=sci_arttext\&pid= S0864-21251999000200011 Acceso el 12 de septiembre de 2017.

18. Cuba, Ministerio de Salud Pública, Departamento Materno Infantil y Planificación Familiar. Programa Nacional de Salud para la Atención Integral en la Adolescencia, 2012-2017. La Habana: MINSAP; 2012. Disponible en: http:// www.codajic.org/sites/www.codajic. org / files / Programa $\% 20$ nacional $\% 20$ de $\% 20$ salud $\% 20$ para $\% 201$ a $\% 20$ atenci $\%$ C3\%B3n\%20integral\%20en\%20la\%20adolescencia\%20\%28Cuba\%29.pdf Acceso el 16 de noviembre de 2017.

19. Robaina Castellanos GR, Riesgo Rodríguez SC. Niños dependientes de tecnología, un problema emergente de salud.
Rev Cubana Pediatr. 2012;84(3):282-92. Disponible en: http://www.medigraphic. com/pdfs / revcubped/cup-2012/cup123i. pdf Acceso el 24 de septiembre de 2017.

20. Román Lafont J, Álvarez Valdés G, Izquierdo Izquierdo ME. Historia de la puericultura en Cuba. Rev Cubana Pediatr. 2017;89(2). Disponible en: http:/ / www.revpediatria.sld.cu/index.php / ped/article/view/225 Acceso el 30 de marzo de 2017.

21. Esquivel M, Álvarez G, Izquierdo ME, Martínez D, Tamayo V. Well Child Care: A comprehensive strategy for Cuban children and adolescents. MEDICC Rev. 2014;16(1):7-11. Disponible en: http:// www.medicc.org/mediccreview/index. php issue $=27 \& i d=338 \& a=$ vahtml Acceso el 7 de agosto de 2017.

22. United Nations Children's Fund. Early childhood development in Cuba. La Habana: UNICEF; 2016. Disponible en: https: / / www.unicef.org / cuba / cu_ resources_earlychildhooddevelopmentlibro.pdf Acceso el 7 de junio de 2017.

23. Drain PK, Barry M. Fifty years of U.S. embargo: Cuba's health outcomes and lessons. Science. 2010;30;328(5978):572-3. Disponible en: http://www.sciencemag. org / cgi / content/full/328/5978/572 Acceso el 24 de septiembre de 2017.

24. Sánchez Gutiérrez A, Gorry C. Immunodiagnostics: The convergence of bioethics and public health. MEDICC Rev. 2013;15(1):7-10. Disponible en: http:// www.medicc.org/mediccreview/index. php issue $=23 \& i d=286 \& a=$ vahtml Acceso el 6 de agosto de 2017.

25. Marcheco Teruel B. Cuba's National Medical Genetics Program. MEDICC Rev. 2009;11(1):11-3. Disponible en: http:// www.medicc.org/mediccreview /index. php? issue $=7 \& i d=71 \& a=$ vahtml Acceso el 19 de septiembre de 2017.

26. Guzmán Rodríguez E, Guzmán Rubin E, Fernández Riverón F. Terapia intensiva. En: de la Torre Montejo E, Pelayo GonzálezPosada EJ, ed. Pediatría. PX TII. La Habana: Editorial Ciencias Médicas; 2006.

27. Casanova R, Selman-Houssein E, Palenzuela H, Cárdenas F, Carballés J, Savio A, et al. Cuba's National Pediatric Cardiology Program. MEDICC Rev. 2010; 12(2):6-9. Disponible en: http:/ / www.medicc.org/mediccreview $/$ index.php?issue= $12 \& \mathrm{id}=138 \& \mathrm{a}=$ vahtml Acceso el 12 de junio de 2017.

28. Gorry C. Cuba's human breast milk banks. MEDICC Rev. 2014;16(1). Disponible en: http:/ / www.medicc.org/mediccreview / index.php?issue $=27 \& i d=339 \& a=$ vahtm Acceso el 12 de junio de 2017.

29. Valdés Lazo F, Clúa Calderín AM, Podadera Valdés X, Zacca E. Encuesta Nacional de Accidentes en menores de 20 años en Cuba. La Habana: Editorial Ciencias Médicas; 2007

30. Cuba, Ministerio de Salud Pública. Programa del Médico y la Enfermera de la Familia. La Habana: Editorial Ciencias Médicas. 2011. Disponible en: http:/ / files. sld.cu/sida/files/2012/01/programa-medicoy-enfermera-2011-vigente.pdf Acceso el 3 de septiembre de 2017.
31. Cuba, Ministerio de Salud Pública, Departamento Nacional de Salud Materno Infantil. Actualización del programa de morbilidad y mortalidad materna. La Habana: MINSAP; 2012.

32. Organización Panamericana de la Salud, Organización Mundial de la Salud. Relatoría General, Reunión Regional para la Estrategia de Vigilancia y Control de las Arbovirosis. Cuba, 2016. La Habana: OPS 2016. Disponible en: http://www.paho. org/cub/index.php?option=com_docman\& view $=$ download\&alias $=1445$-relatoriarrarbovirosis-2016\&category_slug= documentacion-tecnica\&Itemid $=226$ Acceso el 7 de junio de 2017.

33. Cuba, Ministerio de Salud Pública, Dirección de Registros Médicos y Estadísticas de Salud. Encuesta de Indicadores Múltiples por Conglomerados. Cuba, 2014. Informe final. La Habana: Fondo de Naciones Unidas para la Infancia; 2015. Disponible en: https://www.unicef.org/cuba/mics5-2014cuba.pdf Acceso el 7 de junio de 2017.

34. Pita G, Jiménez S, Basabe B, García RG, Macías C, Selva L, et al. Anemia in children under five years old in Eastern Cuba, 2005-2011. MEDICC Rev. 2014;16(1):16-23. Disponible en: http://www.medicc.org/mediccreview / index.php?issue $=27 \& i d=340 \& a=$ vahtml Acceso el 24 de septiembre de 2017.

35. Keck CW, Reed GA. The curious case of Cuba. Am J Pub Health. 2012;102(8) e13-e22. Disponible en: http:/ / ajph.aphapublications.org/doi/pdf/10.2105/AJPH. 2012.300822 Acceso el 24 de septiembre de 2017.

36. Oficina Nacional de Estadística e Informática. Los Objetivos de Desarrollo del Milenio. Cuba. Edición 2015. La Habana: ONEI; 2015. Disponible en: http:/ / www.one.cu/publicaciones / 08informacion/ODM2014.pdf Acceso el 6 de junio de 2017

37. Organización Mundial de la Salud. La OMS valida la eliminación en Cuba de la transmisión de madre a hijo del VIH y de la sífilis [comunicado de prensa], 2015 junio 30. Ginebra: OMS; 2015. Disponible en: http: / / www.who.int/mediacentre / news / releases / 2015/mtct-hiv-cuba/es / Acceso el 4 de octubre de 2017.

38. Fondo de las Naciones Unidas para la Infancia. Los jóvenes y el VIH/sida. Una oportunidad en un momento crucial. París: UNICEF-ONUSIDA-OMS; 2002. Disponible en: https://www.unicef.org/ lac/Los_jovenes_y_el_VIH-SIDA._Una oportunidad_en_un_momento_crucial. pdf Acceso el 3 de junio de 2017.

39. Naciones Unidas. Informe de los Objetivos de Desarrollo Sostenible 2016. Nueva York: NU; 2016. Disponible en: https:// unstats.un.org/sdgs / report/2016/ The $\% 20$ Sustainable $\% 20$ Development $\% 20$ Goals\%20Report\%202016_Spanish.pdf Acceso el 3 de junio de 2017.

Manuscrito recibido el 27 de abril de 2017. Aceptado para publicación, tras revisión, el 9 de noviembre de 2017. 
ABSTRACT In Cuba, maternal and child health care is based on the priority granted by the State, the implementation of the National Maternal and Child Health Program and the guarantee of equitable access to health services. This article describes the Cuban expe-

Maternal and child health care in Cuba: achievements and challenges

Keywords Maternal health; child health; maternal mortality; infant mortality; Millennium

Development Goals; Sustainable Development Goals; Cuba.

rience in this field, as well as its main achievements, challenges, and lessons learned.
Among the most relevant results up to 2015 are the reduction of infant mortality rate and under-five mortality rate to 4.3 and 5.7 deaths per 1000 live births, respectively; 5 -year survival of $99.4 \%$; more than 10 prenatal check-ups per delivery; $5.3 \%$ of low birth weight; $99.9 \%$ of institutional births; and being the first country to validate the elimination of mother-to-child transmission of HIV/AIDS and congenital syphilis. The main challenges are to increase the rate of exclusive breastfeeding; to reduce anemia due to iron deficiency in children and pregnant women; to reduce overweight in children; to prevent accidents; and to reduce maternal mortality, adolescent fertility rate, and voluntary abortion. Among the lessons learned are the priority given by the State to health, the programmatic management of maternal and child care, the guarantee of universal coverage, the systematic collection of information for decision-making, the integration of sectors and social participation in health. Sustaining and improving the results achieved will contribute to the fulfillment of the Sustainable Development Agenda for 2030.
RESUMO

Palavras-chave

\section{Cuidados de saúde materno-infantil em Cuba: conquistas e desafios}

Em Cuba, os cuidados de saúde materno-infantil baseiam-se na prioridade concedida pelo Estado, na implementação do Programa Nacional de Saúde Materno-Infantil e na garantia de acesso equitativo aos serviços de saúde. Este artigo descreve a experiência cubana neste campo, bem como as principais conquistas, desafios e lições aprendidas. Entre os resultados mais relevantes até 2015 estão a redução da mortalidade infantil e mortalidade em menores de 5 anos para 4,3 e 5,7 mortes por 1000 nascidos vivos, respectivamente; sobrevivência a 5 anos de $99,4 \%$; mais de 10 exames pré-natal por nascimento; $5,3 \%$ do baixo peso ao nascer; $99,9 \%$ dos partos institucionais; e seja o primeiro país a validar a eliminação da transmissão materno-infantil de HIV / AIDS e sífilis congênita. Os principais desafios são aumentar a taxa de aleitamento materno exclusivo; reduzir a anemia devido a deficiência de ferro em crianças e mulheres grávidas e reduzir o excesso de peso infantil; prevenir acidentes; e reduzir a mortalidade materna, taxa de fertilidade adolescente e aborto voluntário. Entre as lições aprendidas estão a prioridade dada pelo Estado à saúde, a gestão programática dos cuidados materno e infantil, a garantia de cobertura universal, a coleta sistemática de informações para a tomada de decisões, a integração de setores e a participação social em saúde. Sustentar e melhorar os resultados obtidos contribuirá para o cumprimento da Agenda de Desenvolvimento Sustentável para 2030.

Saúde materna; saúde da criança; mortalidade materna; mortalidade infantil; Objetivos de Desenvolvimento do Milênio; Objetivos de Desenvolvimento Sustentável; Cuba. 